\title{
Poaceae cespitosa e decumbente adubadas com NPK: efeitos na agregação do solo ${ }^{1}$
}

\author{
Dácio Jerônimo de Almeida ${ }^{2}$ Ivandro de França da Silva², \\ Flávio Pereira da Mota Silveira ${ }^{2}$, Robeval Diniz Santiago ${ }^{2}$, José Ronaldo Calado Costa ${ }^{2}$
}

\begin{abstract}
Caespitosa and decumbens Poaceae

fertilized with NPK: effects on soil aggregation

The disintegration of soil particles affects its productive capacity, resulting in compression, erosion and nutrient losses. This study aimed at evaluating the aggregates quality and stability of a Yellow Latosol, in a randomized blocks design represented by Urochloa decumbens and Urochloa brizantha, with and without mineral fertilization, and two sampling forms (rows and between rows), in three sampling depths. The undeformed soil samples collected were shattered and passed through a $9.52 \mathrm{~mm}$ sieve and, after drying, through a $2.00 \mathrm{~mm}, 1.00 \mathrm{~mm}$, $0.50 \mathrm{~mm}$ and $0.25 \mathrm{~mm}$ sieves, according to the following diameters classes: $9.52-2.00 \mathrm{~mm}, 2.00-1.00 \mathrm{~mm}, 1.00-0.50 \mathrm{~mm}$ and $0.50-0.25 \mathrm{~mm}$, by using dry sieving. These aggregates were also separated by wet sieving and the dispersed clay and root dry matter were determined. Aggregates larger than $2.00 \mathrm{~mm}$ showed high stability, in the $0.00-0.05 \mathrm{~m}$ layer, when compared to the aggregates from smaller classes, with no effect of sampling and fertilization site on the aggregates size and stability. There was no difference for the two species analyzed, sampling method and fertilization on the aggregates formation and stability.
\end{abstract}

KEY-WORDS: Urochloa sp.; granulometry; dry matter.

\section{INTRODUÇÃO}

Em decorrência do manejo, a desestruturação, compactação e redução da permeabilidade e dos teores de matéria orgânica do solo são consideradas as principais causas da degradação de solos agrícolas, com consequências negativas ao seu potencial produtivo. Nos solos, quando o tamanho dos agregados é reduzido, o espaço poroso compõe-se de uma maior percentagem de microporos, dificultando a infiltração de água, a penetração das raízes e a aeração do solo (Queiroz et al. 2011).

A agregação do solo é um fenômeno que ocorre com a aproximação e posterior estabilização

\section{RESUMO}

A desagregação de partículas do solo afeta a sua capacidade produtiva, resultando em processos de compactação, erosão e perdas de nutrientes. Neste estudo, foi avaliada a qualidade e estabilidade dos agregados de um Latossolo Amarelo, em delineamento de blocos ao acaso, representado por Urochloa decumbens e Urochloa brizantha, na presença e ausência de adubação mineral, e por duas formas de amostragem (fileiras e entre as fileiras), em três profundidades de amostragem. As amostras indeformadas de solo coletadas foram destorroadas, passadas em peneira de $9,52 \mathrm{~mm}$ e, após secagem, em peneiras de 2,00 mm; $1,00 \mathrm{~mm} ; 0,50 \mathrm{~mm}$ e $0,25 \mathrm{~mm}$, obedecendo às seguintes classes de diâmetro: 9,52-2,00 mm; 2,00-1,00 mm; $1,00-0,50 \mathrm{~mm}$ e $0,50-0,25 \mathrm{~mm}$, por peneiragem seca. Estes agregados foram, também, separados por peneiragem úmida, sendo, ainda, determinada a argila dispersa e a massa seca de raízes. Os agregados maiores que 2,00 $\mathrm{mm}$ apresentaram estabilidade elevada, na camada de 0,00-0,05 m, em comparação aos agregados de menores classes, não havendo efeito do local de amostragem e de adubação sobre o tamanho e estabilidade dos agregados. Concluiu-se não haver diferença entre as duas espécies estudadas, forma de coleta das amostras e adubação, na formação e estabilidade de agregados.

PALAVRAS-CHAVE: Urochloa sp.; granulometria; massa seca.

das partículas, por agentes cimentantes, envolvendo a ação de componentes como o regime hídrico, composição húmica e capacidade de troca de cátions, dentre outros (Bastos et al. 2005).

Estudando a agregação e a estabilidade dos agregados de um Latossolo, no Mato Grosso do Sul, Salton et al. (2008) verificaram que sistemas de manejo do solo com pastagem permanente, ou mesmo em rotação de culturas (lavoura-pecuária), favoreceram a formação de agregados estáveis e de maior tamanho, resultando em melhor desenvolvimento das raízes, infiltração e circulação de água e ar, além de aumento na microfauna do solo.

1. Trabalho recebido em maio/2013 e aceito para publicação em fev./2014 (nº registro: PAT 24108).

2. Universidade Federal da Paraíba (UFPB), Centro de Ciências Agrárias, Departamento de Solos e Engenharia Rural, Areia, PB, Brasil.E-mails: almeida_dacio@hotmail.com, ivandro@cca.ufpb.br, flaviopms@hotmail.com, robevaldiniz@bol.com.br, ronaldo.calado@hotmail.com. 
Kitamura et al. (2008) observaram que Urochloa decumbens, após um ano em Latossolo Vermelho degradado, reduziu a densidade do solo de $1,77 \mathrm{~kg} \mathrm{dm}^{-3}$ para $1,58 \mathrm{~kg} \mathrm{dm}^{-3}$. Esta contribuição ocorreu devido à liberação de exsudatos pelas raízes, como, também, pelo favorecimento no aumento da população de micro-organismos, pois ambos os benefícios exercem atividades agregantes.

Para a obtenção dos benefícios proporcionados pelas Poaceae, são necessários cuidados com o manejo oferecido às plantas, concedendo condições ideais para o seu desenvolvimento, como adubação, calagem, altura de pastejo e controle de plantas daninhas e número de animais por área, dentre outros.

Oliveira et al. (2005), trabalhando com métodos de recuperação de pastagens de Urochloa brizantha cv. Marandu, por meio de adubação e calagem, proporcionaram à Poaceae incremento de matéria seca acima de $6 \mathrm{tha}^{-1}$, em relação ao tratamento testemunha, o que possibilitou maior cobertura do solo, como, também, redução na competição de outras espécies.

Este trabalho objetivou avaliar a qualidade dos agregados de um Latossolo Amarelo, quanto à sua resistência à desintegração em água, cultivado com Poaceaes dos gêneros Urochloa decumbens e Urochloa brizantha (cultivar MG5), coletados nas fileiras e entre as fileiras, na ausência e presença de adubação mineral com NPK, aplicado, superficialmente, em toda a área.

\section{MATERIAL E MÉTODOS}

A pesquisa foi conduzida em condições de campo, de novembro de 2005 a novembro de 2010, em fazenda pertencente ao Centro de Ciências Agrárias da Universidade Federal da Paraíba, em Areia (PB), microrregião do Brejo Paraibano (6 $6^{\circ} 8^{\prime} 12$ "S, $35^{\circ} 41^{\prime} 15^{\prime}$ "W e altitude de $620 \mathrm{~m}$ ). O clima do local é do tipo As', conforme classificação de Köppen, correspondendo a clima tropical, quente e úmido, com chuvas no período de outono e inverno, precipitação pluvial média anual de $1.400 \mathrm{~mm}$ e temperatura média anual de $23^{\circ} \mathrm{C}$ (Gondim 1999). O solo foi classificado como Latossolo Amarelo (Embrapa 2006), apresentando, nos $0,30 \mathrm{~m}$ superficiais, textura argilo-arenosa (areia total $=506 \mathrm{~g} \mathrm{~kg}^{-1}$; silte $=$ $103 \mathrm{~g} \mathrm{~kg}^{-1}$; argila $\left.=391 \mathrm{~g} \mathrm{~kg}^{-1}\right)$.

Aárea experimental foi constituída por delineamento de blocos casualizados, com três repetições, sendo cada bloco representado por duas parcelas, com dimensões de 10,0 m de comprimento por 5,0 m de largura, subdivididas em duas subparcelas de $5,0 \mathrm{~m}$ x 5,0 m, para aplicação dos tratamentos com e sem adubação mineral.

Empregou-se o esquema fatorial $2 \times 2 \times 2$, com medidas repetidas no espaço (três profundidades), representado por duas Poaceaes (Urochloa decumbens e Urochloa brizantha cultivar MG5); duas condições de adubação mineral com NPK (ausência e presença); duas formas de amostragem (nas fileiras e entre as fileiras de plantio das gramíneas), em virtude de a Urochloa decumbens apresentar hábito decumbente e a Urochloa brizantha hábito cespitoso; e três profundidades de amostragem (0,00-0,05 m; 0,05-0,15 m; 0,15-0,30 m).

A adubação consistiu de $553 \mathrm{~kg} \mathrm{ha}^{-1}$ da mistura de NPK (60-80-45), tendo, como fonte de nutrientes, respectivamente, o sulfato de amônio, superfosfato triplo e cloreto de potássio, aplicados, manualmente, a lanço, no início do período chuvoso, após o corte de uniformização das Poaceaes, no ano de 2006, prática repetida anualmente.

Após cinco anos (novembro de 2005 a novembro de 2010) do plantio das Poaceaes, na área experimental, amostras indeformadas foram retiradas, nas subparcelas e nas profundidades estabelecidas, com o auxílio de pá reta de corte, com massa de, aproximadamente, $2,0 \mathrm{~kg}$, sob a linha de plantio e nas entrelinhas.

Em laboratório, as amostras de solo foram destorroadas manualmente, passadas por peneira de 9,52 mm de diâmetro de malha e colocadas para secar ao ar livre, à sombra. Após a secagem das amostras, estas foram passadas em peneiras de 2,00 mm; $1,00 \mathrm{~mm} ; 0,50 \mathrm{~mm}$ e $0,25 \mathrm{~mm}$ de diâmetro e separadas, para obtenção de agregados correspondentes às seguintes classes de tamanho de diâmetro: 9,52-2,00 mm; 2,00-1,00 mm; 1,00-0,50 mm e $0,50-0,25 \mathrm{~mm}$, por meio de peneiragem seca (Silva \& Mielniczuk 1997).

Os agregados separados por classes de diâmetro foram submetidos a umedecimento, por 24 horas, e, após este período, à separação por peneiragem úmida (Tisdall et al. 1978, modificado por Carpenedo \& Mielniczuk 1990), para verificação da desagregação em unidades menores. A estabilidade dos agregados foi determinada por meio da relação entre diâmetro médio ponderado de agregados úmidos e diâmetro médio ponderado de agregados secos (Silva \& Mielniczuk 1997). 
A quantidade de argila dispersa em água foi determinada conforme metodologia descrita pela Embrapa (1997). Para quantificação das raízes, foram retiradas amostras de solo nas fileiras e entre as fileiras de plantio, com o auxílio de placa de pregos $(0,30 \mathrm{~m}$ de comprimento por $0,20 \mathrm{~m}$ de largura e espessura de $0,08 \mathrm{~m}$ ), as quais foram separadas nas três profundidades utilizadas no estudo. As partes fracionadas foram imersas em baldes plásticos contendo água, para desestruturação do material, com a finalidade de separar o solo das raízes, e, em seguida, todo o material foi passado em peneira de $1,00 \mathrm{~mm}$ de malha, para retenção das raízes. Em seguida, foram quantificadas, após acondicionamento em sacos de papel, e colocadas para secagem em estufa com circulação forçada de ar, a $65^{\circ} \mathrm{C}$, até atingirem peso constante, e pesadas, para a obtenção dos valores de matéria seca.

Os dados foram submetidos à análise de variância de medidas repetidas no espaço (profundidade), considerando-se a estrutura de covariância autorregressiva (AR1). As médias das Poaceaes, adubação e formas de amostragem foram comparadas pelo teste F, e as de profundidade pelo teste Tukey.

\section{RESULTADOS E DISCUSSÃO}

Nos resultados de diâmetro médio ponderado dos agregados obtidos por peneiragem seca (DMPAs) e úmida (DMPAu), verificou-se que não houve diferenças significativas. Apesar disto, os valores de DMPAs e DMPAu foram maiores na camada superficial $(0,00-0,05 \mathrm{~m})$, em relação às duas outras camadas (Tabela 1).
De maneira geral, os valores tendem a diminuir com a profundidade avaliada, em razão da maior concentração de raízes nos primeiros centímetros do perfil do solo, contribuindo para maior atividade dos agentes cimentantes, como o crescimento e funcionamento das raízes, atividade dos micro-organismos do solo e exsudação radicular. Salton et al. (2008), em experimentos de longa duração, com diferentes sistemas de manejo, verificaram que sistemas com pastagem apresentaram maior diâmetro médio ponderado de agregados, constatando redução nos valores, à medida em que aumentava a profundidade avaliada.

Mesmo não tendo sido alterada pelos tratamentos estudados, verificou-se que a estabilidade dos agregados apresentou relação direta com a massa seca de raízes. Já com relação às profundidades avaliadas, os maiores valores de DMPAs e DMPAu encontrados na camada superficial do solo estão relacionados, diretamente, com o maior valor de massa seca de raízes, que se diferencia das demais camadas. Nas camadas de 0,05-0,15 m e 0,15-0,30 m, não ocorreram diferenças estatísticas, exceto para as raízes.

Apesar de existirem diferenças para a massa seca de raízes, quando comparadas as duas formas de coleta do solo, na fileira e entre as fileiras de plantio, estas não influenciaram na formação e estabilidade dos agregados, para as mesmas condições (Tabela 1). O mesmo foi verificado para a ausência e presença de adubação mineral com NPK, apesar de a adubação ter promovido incremento significativo na produção de fitomassa seca de raízes (Tabela 1).

Eshel \& Waisel (1996) afirmam que o estado nutricional das plantas tem efeito nas propriedades

Tabela 1. Valores de diâmetro médio ponderado de agregados secos (DMPAs) e úmidos (DMPAu) e da relação DMPAu/DMPAs, para os diferentes tratamentos avaliados (Areia, PB, 2010).

\begin{tabular}{|c|c|c|c|c|c|}
\hline \multirow{4}{*}{ Tratamento } & \multicolumn{5}{|c|}{ Determinações } \\
\hline & \multicolumn{2}{|c|}{ Agregados } & \multirow{3}{*}{$\begin{array}{c}\text { Estabilidade } \\
\text { DMPAu/DMPAs }\end{array}$} & \multirow{2}{*}{ Argila dispersa } & \multirow{2}{*}{$\begin{array}{c}\text { Massa seca de } \\
\text { raízes }\end{array}$} \\
\hline & DMPAs & DMPAu & & & \\
\hline & \multicolumn{2}{|c|}{$\mathrm{mm}$} & & $\%$ & $\mathrm{~g}$ \\
\hline U. brizantha & 2,982 & 2,381 & 0,798 & 37 & 7,64 \\
\hline U. decumbens & 3,018 & 2,385 & 0,790 & 40 & 6,80 \\
\hline Com adubação & 3,028 & 2,413 & 0,797 & 40 & $8,70 \mathrm{a}$ \\
\hline Sem adubação & 2,972 & 2,352 & 0,791 & 37 & $5,73 \mathrm{~b}$ \\
\hline Na fileira & 2,971 & 2,375 & 0,799 & 39 & $9,02 \mathrm{a}$ \\
\hline Entre as fileiras & 3,028 & 2,391 & 0,790 & 39 & $5,41 \mathrm{~b}$ \\
\hline $0,00-0,05 \mathrm{~m}$ & 3,253 & 2,627 & 0,808 & 39 & $11,94 \mathrm{a}$ \\
\hline $0,05-0,15 \mathrm{~m}$ & 2,886 & 2,272 & 0,787 & 40 & $11,57 \mathrm{a}$ \\
\hline $0,15-0,30 \mathrm{~m}$ & 2,859 & 2,249 & 0,787 & 37 & $6,15 b$ \\
\hline
\end{tabular}

Médias seguidas da mesma letra minúscula na coluna, dentro de cada tratamento, não diferem pelo teste Tukey, a $5 \%$. 
de crescimento de suas raízes, entretanto, este incremento não foi suficiente para proporcionar diferença na agregação e na estabilidade dos agregados.

Os baixos valores de argila dispersa em água refletem na maior estabilidade dos agregados, em consequência da presença das Poaceaes na área estudada (Tabela 1). Silva \& Mielniczuk (1997) relacionam a presença de gramíneas com maior aporte de raízes à maior agregação das partículas do solo, tanto pelo efeito mecânico, como, provavelmente, pela decomposição da matéria orgânica, além da liberação de exsudatos.

Observou-se, às profundidades de $0,00-0,05 \mathrm{~m}$; $0,05-0,15 \mathrm{~m}$; e $0,15-0,30 \mathrm{~m}$, que os agregados com diâmetro superior a 2,00 $\mathrm{mm}$, após peneiragem úmida, apresentaram maior resistência à desagregação que as outras classes de diâmetro, com valores médios, respectivamente, de $48,5 \%$; $45,2 \%$; e $42,6 \%$, seguidos das classes de diâmetro de 1,00-0,50 mm (45,8\%; $44,0 \%$; e $38,0 \%)$ e $2,00-1,00 \mathrm{~mm}(36,8 \% ; 37,8 \%$; e $35,3 \%$ ), com menor valor para a classe de diâmetro de 0,50-0,25 mm (33,1\%; 34,2\%; e 29,6\%) (Tabela 2).

Os resultados para agregados de diâmetro entre 2,00 $\mathrm{mm}$ e $1,00 \mathrm{~mm}$ evidenciaram que os percentuais de estabilidade diminuíram, atingindo valores próximos a $40 \%$, para as duas Poaceaes. A presença de adubação, nas duas formas de amostragem, não resultou em diferença significativa entre Urochloa decumbens (34,3\%) e Urochloa brizantha MG5 (38,9\%), devido ao fato de as raízes fasciculadas das Poaceaes não se absterem apenas na linha de plantio, além de os adubos sulfato de amônio e superfosfato triplo fornecerem nutrientes como o nitrogênio e o fósforo, responsáveis pelo desenvolvimento e crescimento da parte vegetativa, aumentando o vigor da rebrota e o número de perfilhos (Alexandrino et al. 2004) e melhorando a liberação de exsudatos, os quais possuem efeito agregante nas partículas do solo.

Já para os agregados com diâmetro entre $1,00 \mathrm{~mm}$ e $0,50 \mathrm{~mm}$, estes apresentaram melhor estabilidade, em relação ao diâmetro entre $2,00 \mathrm{~mm}$ e 1,00 mm. Neste diâmetro, a estabilidade subiu para uma média de $42,6 \%$, com valores que atingiram $54,5 \%$, confirmando as constatações de Albuquerque et al. (1995) e Costa et al. (2003), de que agregados menores são mais resistentes à desagregação do que os maiores. A Urochloa brizantha MG5 apresentou valor médio de $44,5 \%$, enquanto, para a Urochloa decumbens, este valor foi de $40,7 \%$.
A percentagem de agregados com diâmetro de 0,50-0,25 mm, com valores inferiores às demais classes de agregados, corroboram os resultados encontrados por Pikul et al. (2009), que, em pesquisa de campo avaliando a matéria orgânica e a estabilidade dos agregados em água, em sistema de plantio direto e convencional, em rotações de milho e soja, concluíram, após quatro anos, que a estabilidade dos agregados em água aumentou com o tamanho dos agregados, nestes sistemas de cultivos.

A partir dos valores de distribuição percentual dos agregados, às profundidades de 0,00-0,05 m; 0,05-0,15 m; e 0,15-0,30 m (Tabela 2), observou-se que os agregados maiores que 2,00 $\mathrm{mm}$ apresentaram estabilidade elevada, com média para agregados próxima de $50 \%$; 45,0\%; e 43,0\%, respectivamente. Atingiram, inclusive, 52,6\%, para o tratamento com Urochloa brizantha MG5 não adubado, com agregados amostrados entre as fileiras da Poaceae, à profundidade de 0,00-0,05 m; de 49,2\%, para o tratamento com Urochloa decumbens não adubado, amostrado nas fileiras de plantio, para a profundidade de $0,05-0,15 \mathrm{~m}$; e de 48,9\%, para o tratamento com Urochloa brizantha MG5 não adubado, amostrado entre as fileiras da Poaceae, à profundidade de 0,15-0,30 $\mathrm{m}$.

Verificou-se, ainda, que, com o aumento da profundidade, a estabilidade regrediu para as duas Poaceaes, inclusive nas duas formas de amostragem. Isto, provavelmente, deveu-se ao fato de o crescimento das raízes se concentrar nas camadas mais superficiais, bem como à própria morfologia radicular, pois as Poaceaes, mesmo tendo hábito de crescimento decumbente e cespitoso, apresentam caráter fasciculado. Além disto, as camadas superficiais estão expostas de forma mais intensa aos ciclos de umedecimento e secagem, contribuindo para a estabilidade dos agregados (Wendling et al. 2005).

Observou-se não haver diferenças entre Urochloa brizantha MG5 e Urochloa decumbens, na ausência e presença de adubação mineral com NPK, ocorrendo uma pequena diferença nas formas de coleta das amostras, na qual as coletadas nas fileiras de plantio se destacaram, por apresentarem maior concentração de raízes, em relação àquelas coletadas entre as fileiras (Tabela 1).

O maior valor de estabilidade de agregados foi observado para as amostras coletadas entre as fileiras de gramíneas, como, também, para os tratamentos sem adubação mineral (Tabela 2). Estes valores ligeiramente maiores ocorreram devido à expansão 
Tabela 2. Distribuição de agregados em diferentes classes de diâmetro (Areia, PB, 2010).

\begin{tabular}{|c|c|c|c|c|}
\hline \multirow{4}{*}{ Gramínea } & \multicolumn{4}{|c|}{ Agregados } \\
\hline & \multicolumn{2}{|c|}{ Com adubação } & \multicolumn{2}{|c|}{ Sem adubação } \\
\hline & Na fileira & Entre as fileiras & Na fileira & Entre as fileiras \\
\hline & \multicolumn{4}{|c|}{$\%$} \\
\hline \multicolumn{5}{|c|}{ Profundidade de 0,00-0,05 m } \\
\hline \multicolumn{5}{|c|}{ Agregados com diâmetro entre $9,52 \mathrm{~mm}$ e $2,00 \mathrm{~mm}$} \\
\hline U. decumbens & 51,2 & 42,3 & 51,6 & 45,3 \\
\hline U. brizantha & 48,5 & 50,1 & 46,6 & 52,6 \\
\hline \multicolumn{5}{|c|}{ Agregados com diâmetro entre $2,00 \mathrm{~mm}$ e $1,00 \mathrm{~mm}$} \\
\hline U. decumbens & $33,4 \mathrm{bA} \alpha$ & $25,6 \mathrm{bBB}$ & $30,8 \mathrm{~B}$ & $38,6 \alpha$ \\
\hline U. brizantha & $41,0 \mathrm{a}$ & $44,4 \mathrm{a}$ & 36,6 & 43,8 \\
\hline \multicolumn{5}{|c|}{ Agregados com diâmetro entre $1,00 \mathrm{~mm}$ e $0,50 \mathrm{~mm}$} \\
\hline U. decumbens & $46,3 \mathrm{~A}$ & $36,8 \mathrm{bBB}$ & 42,4 & $45,9 \mathrm{~b} \alpha$ \\
\hline U. brizantha & $48,5 \alpha$ & $47,7 \mathrm{a}$ & $44,3 \mathrm{~B}$ & 54,5 a \\
\hline \multicolumn{5}{|c|}{ Agregados com diâmetro entre $0,50 \mathrm{~mm}$ e $0,25 \mathrm{~mm}$} \\
\hline U. decumbens & 35,5 & $31,2 \mathrm{~b}$ & 31,6 & 34,0 \\
\hline U. brizantha & 34,9 & $44,9 \mathrm{a}$ & 33,8 & 40,9 \\
\hline \multicolumn{5}{|c|}{ Profundidade de 0,05-0,15 m } \\
\hline \multicolumn{5}{|c|}{ Agregados com diâmetro entre $9,52 \mathrm{~mm}$ e $2,00 \mathrm{~mm}$} \\
\hline U. decumbens & 45,7 & 42,1 & 49,2 & 46,9 \\
\hline U. brizantha & 45,7 & 45,4 & 41,9 & 44,8 \\
\hline \multicolumn{5}{|c|}{ Agregados com diâmetro entre $2,00 \mathrm{~mm}$ e $1,00 \mathrm{~mm}$} \\
\hline U. decumbens & $39,9 \mathrm{~A} \alpha$ & $32,6 \mathrm{~B}$ & $34,3 \mathrm{~B}$ & 42,9 \\
\hline U. brizantha & $41,7 \mathrm{~A} \alpha$ & $35,9 \mathrm{~B}$ & $31,7 \mathrm{BB}$ & $43,5 \mathrm{~A}$ \\
\hline \multicolumn{5}{|c|}{ Agregados com diâmetro entre $1,00 \mathrm{~mm}$ e $0,50 \mathrm{~mm}$} \\
\hline U. decumbens & 45,2 & 41,1 & 42,9 & $43,3 \mathrm{~b}$ \\
\hline U. brizantha & $47,5 \mathrm{~A} \alpha$ & $40,7 \mathrm{~B}$ & $41,4 \mathrm{~B}$ & $51,3 \mathrm{a}$ \\
\hline \multicolumn{5}{|c|}{ Agregados com diâmetro entre $0,50 \mathrm{~mm}$ e $0,25 \mathrm{~mm}$} \\
\hline U. decumbens & 35,2 & 31,6 & 32,6 & 34,6 \\
\hline U. brizantha & 36,6 & 34,0 & 32,3 & 36,4 \\
\hline \multicolumn{5}{|c|}{ Profundidade de $0,15-0,30 \mathrm{~m}$} \\
\hline \multicolumn{5}{|c|}{ Agregados com diâmetro entre $9,52 \mathrm{~mm}$ e $2,00 \mathrm{~mm}$} \\
\hline U. decumbens & 42,0 & 42,9 & 39,4 & 42,2 \\
\hline U. brizantha & $37,0 \mathrm{~B}$ & $43,6 \mathrm{~A}$ & 48,9 & 45,4 \\
\hline \multicolumn{5}{|c|}{ Agregados com diâmetro entre $2,00 \mathrm{~mm}$ e $1,00 \mathrm{~mm}$} \\
\hline U. decumbens & 36,1 & 31,2 & 30,9 & $35,5 \mathrm{~b}$ \\
\hline U. brizantha & $31,8 \mathrm{~A} \alpha$ & $35,1 \mathrm{~B}$ & $34,8 \mathrm{~B}$ & $47,0 \mathrm{a}$ \\
\hline \multicolumn{5}{|c|}{ Agregados com diâmetro entre $1,00 \mathrm{~mm}$ e $0,50 \mathrm{~mm}$} \\
\hline U. decumbens & 34,3 & 39,0 & 33,8 & $37,7 \mathrm{~b}$ \\
\hline U. brizantha & $33,2 \mathrm{~A} \alpha$ & $38,2 \mathrm{~B}$ & $37,3 \mathrm{~B}$ & $50,8 \mathrm{a}$ \\
\hline \multicolumn{5}{|c|}{ Agregados com diâmetro entre $0,50 \mathrm{~mm}$ e $0,25 \mathrm{~mm}$} \\
\hline U. decumbens & 23,1 & 30,7 & 25,3 & $30,3 \mathrm{~b}$ \\
\hline U. brizantha & $25,5 \alpha$ & 32,2 & $27,2 \mathrm{~B}$ & $41,9 \mathrm{a}$ \\
\hline
\end{tabular}

Médias seguidas da mesma letra minúscula, na coluna, maiúscula (adubação em formas de amostragem) e grega (formas de amostragem em adubação), na linha, não diferem pelo teste Tukey, a 5\%.

das raízes, na busca pela absorção de nutrientes e água, não se concentrando apenas "abaixo" da planta, o que, consequentemente, acaba explorando maior volume de solo.

Mielniczuk (1996), estudando o desenvolvimento das raízes, de acordo com as práticas de manejo do solo, destacou o efeito radicular da Urochloa brizantha, por ultrapassar camadas compactadas do solo, expandindo, assim, suas raízes, e garantindo a manutenção da produção de fitomassa.

\section{CONCLUSÕES}

1. Não houve diferença entre as duas gramíneas analisadas, tanto na formação como na estabilidade dos agregados. 
2. A coleta de amostras nas fileiras e entre as fileiras de plantio não influenciou na quantidade e na qualidade dos agregados.

3. A adubação mineral com NPK não afetou o tamanho e a estabilidade dos agregados do solo sob pastagem.

4. Os agregados maiores que $2,00 \mathrm{~mm}$ apresentaram maior estabilidade, sendo os maiores valores observados na camada de 0,00-0,05 m.

\section{REFERÊNCIAS}

ALBUQUERQUE, J. A. et al. Rotação de culturas e sistemas de manejo do solo: efeito sobre a forma da estrutura do solo ao final de sete anos. Revista Brasileira de Ciência do Solo, Campinas, v. 19, n. 1, p. 115-119, 1995.

ALEXANDRINO, E. et al. Características morfogênicas e estruturais na rebrotação da Brachiaria brizantha cv. Marandu submetida a três doses de nitrogênio. Revista Brasileira de Zootecnia, Viçosa, v. 33, n. 6, p. 1372-1379, 2004.

BASTOS, R. S. et al. Formação e estabilização de agregados do solo decorrentes da adição de compostos orgânicos com diferentes características hidrofóbicas. Revista Brasileira de Ciência do Solo, Viçosa, v. 29, n. 1, p. 11-20, 2005.

CARPENEDO, V.; MIELNICZUK, J. Estado de agregação e qualidade de agregados de Latossolos Roxos, submetidos a diferentes sistemas de manejo. Revista Brasileira de Ciência do Solo, Campinas, v. 14, n. 1, p. 99-105, 1990.

COSTA, F. S. et al. Propriedades físicas de um Latossolo Bruno afetadas pelos sistemas de plantio direto e preparo convencional. Revista Brasileira de Ciência do Solo, Viçosa, v. 27, n. 3, p. 527-535, 2003.

EMPRESA BRASILEIRA DE PESQUISA AGROPECUÁRIA (Embrapa). Centro Nacional de Pesquisa de Solos. Sistema brasileiro de classificação de solos. Brasília, DF: Embrapa Produção de Informação; Rio de Janeiro: Embrapa Solos, 2006.

EMPRESA BRASILEIRA DE PESQUISA AGROPECUÁRIA (Embrapa). Manual de métodos de análises de solo. Rio de Janeiro: Embrapa Solos, 1997.

ESHEL, A.; WAISEL, Y. Multiform and multifunction of various constituents of one root system. In: WAISEL, Y.; ESHEL, A.; KAFKAFI, U. (Eds.). Plant roots: the hidden half. 2. ed. New York: Marcel Dekker, 1996. p. 175-192.

GONDIM, A. W. de A. Geoeconomia e agricultura do brejo paraibano: análise e avaliação. João Pessoa: UFPB, 1999.
KITAMURA, A. E. et al. Recuperação de solo degradado com aplicação de adubos verdes e de lodo de esgoto. Revista Brasileira de Ciência do Solo, Viçosa, v. 32, n. 1, p. 405-416, 2008.

MIELNICZUK, J. Desenvolvimento de raízes, como método de avaliação das práticas de manejo do solo. In: CASTRO FILHO, C.; MUZILLI, O. (Eds.). Manejo integrado de solos em microbacias hidrográficas. Londrina: Iapar/SBCS, 1996. p. 219-224.

OLIVEIRA, P. P. A. et al. Fertilização com N e S na recuperação de pastagem de Brachiaria brizantha cv. Marandu em Neossolo Quartzarênico. Revista Brasileira de Zootecnia, Viçosa, v. 34, n. 4, p. 1121-1129, 2005.

PIKUL, J. L. et al. Organic matter and water stability of field aggregates affected by tillage in South Dakota. Soil Science Society of America Journal, Madison, v. 73, n. 1, p. 197-206, 2009.

QUEIROZ, R. P. et al. Inter-relation between soybean yield and soil compaction under degraded pasture in Brazilian Savannah. Revista Brasileira de Ciência do Solo, Viçosa, v. 35, n. 5, p. 1579-1588, 2011.

SALTON, J. C. et al. Agregação e estabilidade de agregados do solo em sistemas agropecuários em Mato Grosso do Sul. Revista Brasileira de Ciência do Solo, Viçosa, v. 32, n. 1, p. 11-21, 2008.

SILVA, I. F. da; MIELNICZUK, J. Ação do sistema radicular de plantas na formação e estabilização de agregados do solo. Revista Brasileira de Ciência do Solo, Viçosa, v. 21, n. 1, p. 113-117, 1997.

TISDALL, J. M.; COCKROFT, B.; UREN, N. C. The stability of soil aggregates as affected by organic materials, microbial activity and physical disruption. Australian Journal of Soil Research, Collingwood, v. 16, n. 1, p. 9-17, 1978.

WENDLING, B. et al. Carbono orgânico e estabilidade de agregados de um Latossolo Vermelho sob diferentes manejos. Pesquisa Agropecuária Brasileira, Brasília, DF, v. 40, n. 5, p. 487-494, 2005. 